\title{
Caracterização de topologia de Redes Veiculares baseada em Teoria da Informação
}

\author{
Cristopher G. S. Freitas ${ }^{1}$, Heitor S. Ramos $^{1}$, Raquel S. Cabral ${ }^{3}$, Osvaldo A. Rosso ${ }^{2}$, \\ André L. L. Aquino ${ }^{1}$ \\ ${ }^{1}$ Instituto de Computação, Universidade Federal de Alagoas \\ ${ }^{2}$ Instituto de Física, Universidade Federal de Alagoas \\ ${ }^{3}$ Curso de Ciência da Computação, Arapiraca, Universidade Federal de Alagoas \\ cgsf@ic.ufal.br, heitor@laccan.ufal.br, raquel.cabral@gmail.com \\ oarosso@gmail.com, alla@laccan.ufal.br
}

\begin{abstract}
Vehicular networks can be studied using vehicle's behavior individually varying with time, characterized by displacement or velocities. However, on this work we analyze the aggregated graph-based representation, which describes a global aspect of the network, encapsulating the dynamics of each vehicle during a sampled period, thus, verifying its structural behavior with Information Theory quantifiers for mapping these data onto a Complexity-Entropy plane. This method was applied to 17 vehicular networks, varying synthetically its topologies in V2V, V2I and V2V2I, such way its graphs presented a variable dynamic between Watts-Strogatz and Barabási-Albert models behaviors.
\end{abstract}

Resumo. Redes Veiculares podem ser estudadas utilizando o comportamento individual de cada veículo em relação ao tempo, caracterizados pelo deslocamento ou velocidade. No entanto, neste trabalho iremos analisar o comportamento do grafo agregado, que descreve a rede em um aspecto global, encapsulando toda a dinâmica dos veículos durante o intervalo total amostrado, assim, verificando seus aspectos estruturais com quantificadores de Teoria da Informação para mapear esses dados no plano Complexidade-Entropia. Este método foi aplicado à 17 redes veiculares, variando suas topologias em V2V, V2I e V2V2I, de forma que seus grafos agregados apresentaram uma dinâmica variável entre o comportamento dos modelos Watts-Strogatz e Barabási-Albert.

\section{Introdução}

As Redes Veiculares podem ser representadas por um grafo $G(V, E)$, em que os veículos ou infraestruturas representam um nó $v_{i} \in V$, e cada nó possui um equipamento de comunicação sem fio que ao conectar-se com outro veículo, constitui uma aresta $e_{i} \in E$. O objetivo de uma VANET (Vehicular Ad-hoc Network) é manter a comunicação entre veículos tornando a interação entre eles possível, nesse caso, cada veículo é capaz de enviar e receber dados na rede [Yousefi et al. 2006]. Existem diferentes arquiteturas para a comunicação entre veículos em uma VANET [Liang et al. 2015], dentre elas, temos V2V, V2I e V2V2I. Em uma rede V2V (Vehicle-To-Vehicle) os veículos comunicam-se entre si, sem a necessidade de um intermediário; em uma abordagem V2I (Vehicle-ToInfrastructure) existe uma infraestrutura responsável por entrega e recebimento das men- 
sagens entre veículos; e na abordagem híbrida, denominada V2V2I (Vehicle-To-VehicleTo-Infrastructure), os nós comunicam-se tanto entre si quanto com as infraestruturas disponíveis.

Essas redes são amplamente estudadas com o objetivo de otimizar a conectividade nas vias ao redor do mundo [Hajlaoui et al. 2016], de forma que a disseminação de informação pode ajudar nos aspectos de segurança e eficiência no trânsito. Com isso, entender as características da topologia da Rede Veicular é muito relevante para a escolha de qual abordagem de roteamento utilizar na rede [Liu et al. 2016], caso os veículos tenham informações da posição dos demais nós, pode-se variar o protocolo de roteamento visando melhorar o processo de disseminação de dados de acordo com a dinâmica atual da rede [Zhang et al. 2013].

Redes Veiculares em geral são muito dinâmicas, variando sua topologia constantemente ao logo do tempo, assim, alguns estudos caracterizam a dinâmica dessas redes em busca de identificar padrões de comportamento que possam ajudar na tomada de decisões para projetar e manter essas redes; [Aquino et al. 2015] caracterizam o comportamento de cada veículo individualmente, utilizando séries temporais de velocidade com o método proposto por [Rosso et al. 2007], verificando a variação das velocidades em diferentes intervalos de amostragem; [Tang et al. 2016, Yan et al. 2017] caracterizam a dinâmica do fluxo de tráfego multivariado baseado nas séries temporais de deslocamento, utilizando a teoria de Redes Complexas, analisando métricas como coeficiente de agrupamento e distribuição do grau, identificando os limites onde há transição no espaço de fase, de forma que o tráfego muda de livre para congestionado ao decorrer do dia; [Pappalardo et al. 2013] estudam os padrões de mobilidade humana com carros utilizando séries temporais de deslocamento, avaliando o comportamento local de cada veículo de acordo com as leis de mobilidade, introduzindo métodos para monitoramento de tráfego em tempo real.

Neste trabalho, considera-se o aspecto global do grafo agregado da rede veicular, utilizando a teoria de Redes Complexas para caracterizar a estrutura e dinâmica da rede baseada em sua topologia. O grafo agregado representa todas as conexões realizadas com um veículo em um intervalo de tempo amostrado, onde cada conexão torna-se uma aresta que persiste por todo intervalo, criando uma representação global da rede veicular. Neste sentido, o problema tratado neste trabalho é:

Qual comportamento de uma rede veicular, modelada como um grafo agregado, ao avaliarmos a estrutura da rede e dinâmica dos nós usando as conexões V2V, V2I e V2V2I?

A análise das redes utilizadas neste trabalho será composta principalmente por dois quantificadores de Teoria da Informação: Entropia de Shannon $\boldsymbol{S}$ e a Complexidade Estatística $\boldsymbol{C}$, de forma que suas aplicações levarão em consideração uma distribuição de probabilidade $\boldsymbol{P}$ denominada Random Walk Betweeness [Newman 2005], utilizada para caracterizar as redes reais e o modelo de referência [Erdos 1959] no cálculo do divergente descrito na Seção 2.4. $\boldsymbol{S}$ é capaz de medir a desordem em um sistema, já $\boldsymbol{C}$ quantifica a estrutura do conteúdo deste sistema, onde podemos aplicar esses quantificadores à Redes Complexas utilizando o método proposto por [Wiedermann et al. 2017]. 
Tabela 1: Redes Veiculares utilizadas para caracterização em sua topologia V2V. Sendo $N$ o número de nós, a probabilidade de conexão $\rho$, Entropia da Rede $S$ e a Complexidade Estatística $C$.

\begin{tabular}{lllll}
\hline Descrição da Rede Veicular & $N$ & $\rho$ & $S$ & $C$ \\
\hline Anaheim & 416 & 0.007 & 0.172 & 0.166 \\
Austin & 7387 & 0.000 & 0.112 & 0.056 \\
Barcelona & 930 & 0.004 & 0.191 & 0.188 \\
Berlin-Center & 12981 & 0.000 & 0.119 & 0.059 \\
Berlin-Friedrichshain & 224 & 0.015 & 0.210 & 0.203 \\
Berlin-Mitte-Center & 397 & 0.008 & 0.184 & 0.178 \\
Berlin-Mitte-Prenz-Fried-Center & 974 & 0.003 & 0.164 & 0.159 \\
Berlin-Prenzlauerberg-Center & 352 & 0.009 & 0.182 & 0.176 \\
Berlin-Tiergarten & 359 & 0.009 & 0.194 & 0.189 \\
Chicago-Regional & 12979 & 0.000 & 0.112 & 0.056 \\
Chicago-Sketch & 933 & 0.003 & 0.129 & 0.125 \\
Eastern-Massachusetts & 72 & 0.049 & 0.261 & 0.248 \\
Hessen-Asymmetric & 4660 & 0.001 & 0.103 & 0.103 \\
Philadelphia & 13389 & 0.000 & 0.120 & 0.060 \\
Terrassa-Asymmetric & 1603 & 0.002 & 0.137 & 0.134 \\
Winnipeg-Asymmetric & 948 & 0.003 & 0.148 & 0.144 \\
Winnipeg & 1040 & 0.003 & 0.154 & 0.150 \\
\hline
\end{tabular}

Com essas ferramentas, buscamos caracterizar as topologias de Redes Veiculares V2V, V2I e V2V2I, de acordo com seu comportamento apresentado no plano Complexidade-Entropia. Aliando métricas de Redes Complexas, tais como coeficiente de agrupamento e distância média para garantir a robustez do modelo quanto sua capacidade de caracterizar as redes reais. As demais seções deste trabalho, organizam-se em: Seção 2 que está subdivida em Seção 2.1 que descreve as redes utilizadas no trabalho, Seção 2.2 discute a formalidade para definição das redes e Seção2.3 e 2.4 apresentando os quantificadores e sua metodologia de utilização. Seção 3 discute os resultados e a Seção 4 encerra com as devidas conclusões.

\section{Materiais e Métodos}

\subsection{Base de dados}

Neste trabalho utilizamos 17 redes reais com dados já pré-processados para caracterização das topologias, todas essas redes estão disponíveis no repositório criado pelo grupo $R e$ search Core Team 1 . Cada base possui dados sobre o fluxo da rede, viagens individuais de cada veículo e seu grafo agregado no período amostrado. Consideramos que essas bases, por apresentarem os dados individuais dos nós veículos, seguem uma organização V2V. Na tabela 1 estão os detalhes sobre a quantidade de nós, arestas e probabilidade de conexão $\rho$ de cada rede.

\subsection{Definições das Redes}

Para cada rede consideramos um grafo $G(V, E)$ representado por uma matriz de adjacência $\mathbf{A}$ com dimensões $N \times N$, sendo $N$ o número de nós presentes na rede, onde

1https://github.com/bstabler/TransportationNetworks 
$A_{i j}=1$ caso exista uma conexão entre os nós $i$ e $j$, caso contrário, $A_{i j}=0$. Utilizando um grafo sem laços, não direcionado e sem pesos, temos que a diagonal principal da matriz, $A_{i i}=0 \forall i, \ldots, N, \mathbf{A}=\mathbf{A}^{T}$. O grau de cada nó $k_{i}$ pode ser calculado com $k_{i}=\sum_{n=j}^{N} A_{i j}, k_{i} \leq N-1$. Para nossa análise com Entropia e Complexidade de acordo com a abordagem de [Wiedermann et al. 2017], utilizamos uma distribuição uniforme $P_{i}$ denominada Random Walk Betweenness [Newman 2005], tal que:

$$
p_{i \mapsto j}=\frac{A_{i j}}{k_{i}} \in\left\{0,1 / k_{i}\right\},
$$

sendo $p_{i \mapsto j}$ a probabilidade do nó $i$ saltar aleatoriamente para o nó $j$.

O Betweeness Centrality mede a centralidade de um nó na rede, verificando quantos caminhos mínimos entre um par de nós na rede transversam um nó $i$. Já o Random Walk Betweenness dá ênfase aos caminhos aleatórios que transversam um nó, verificando quão frequente dois outros nós transitam por ele.

\subsection{Entropia da Rede}

A Entropia da Rede $S$ é calculada com a Entropia clássica de Shannon para distribuições discretas:

$$
S(P)=-\sum_{k} p_{k} \log p_{k}
$$

A Entropia clássica de Shannon mede a desordem de um sistema, considerando a probabilidade do sistema apresentar um estado $k$. Levando em consideração a distribuição de probabilidade utilizada (1), para cada nó $i$ temos uma distribuição de probabilidade $P_{i}$, consequentemente teremos uma Entropia para cada nó que será $S_{i}$, dada por

$$
S_{i}\left(P_{i}\right)=-\sum_{j} \frac{A_{i j}}{k_{i}} \log \frac{A_{i j}}{k_{i}}=\log k_{i}
$$

No caso de um nó isolado $i$ com grau $k_{i}=0$, define-se que $S_{i}=0$. Após calculada a Entropia de Shannon para cada nó, encontra-se a média normalizada que caracteriza $S$ :

$$
S(P)=\frac{1}{N \log (N-1)} \sum_{i} S_{i}\left(P_{i}\right) \in[0,1]
$$

A Entropia $S$ pode ser interpretada de acordo com as características Random Walk Betweenness. Para uma rede totalmente conectada, onde $p_{i \mapsto j}=\frac{1}{N-1} \forall i \neq j$, os caminhos tornam-se totalmente aleatórios, assim o nó $i$ pode saltar para qualquer nó $j$, consequentemente a Entropia é maximizada e temos que $S(P)=1$. Em uma rede esparsa, o caminho torna-se mais determinístico por limitar as possibilidades, de maneira que podemos prever com maior frequência para qual nó $j$ será realizado o salto. Assim, quanto mais esparsa for a rede, menor será a Entropia, assim, $S \rightarrow 0$. 


\subsection{Complexidade Estatística}

Em busca de compreender a estrutura física do sistema complexo, a Complexidade Estatística $\boldsymbol{C}$ mede o desequilíbrio do sistema baseado no conteúdo da informação descrita pela distribuição de probabilidade $\boldsymbol{P}$ e sua Entropia $\boldsymbol{S}$ [Lopez-Ruiz et al. 1995]. O desequilíbrio do sistema na mecânica estatística é medido através do divergente de JensonShannon

$$
Q_{i}\left(P_{i}, P_{i, e}\right)=Q_{0}\left\{S_{i}\left(0.5\left[P_{i}+P_{i, e}\right]-0.5\left[S_{i}\left(P_{i}\right)+S_{i}\left(P_{i, e}\right)\right]\right\}\right.
$$

$\operatorname{com} Q_{0}=1 / \log 2$ para garantir $Q_{i} \in[0,1]$. A distribuição $P_{i, e}$ denota a mesma que $P_{i}$ para uma apropriada referência ou estado de equilíbrio, neste caso, uma rede. Nesta abordagem, assume-se que o estado de equilíbrio do sistema corresponde ao totalmente aleatório.

Para uma rede complexa, utilizaremos o modelo Erdós-Rényi como referência para descrever o estado totalmente aleatório. Para satisfazer a equação 5, é necessário comparar as redes reais aos modelos aleatórios, ou seja, calcular $P_{i, e}$. Com o objetivo de garantir a aleatoriedade, para cada rede real utilizada são geradas 100 redes usando o modelo de Erdốs-Rényi, com o mesmo número de nós $N$ e probabilidade de conexão $\rho=\sum_{i} k_{i} /(N(N-1))$, assim, para cada rede de referência criada, computamos $P_{i, e} \mathrm{e}$ $Q_{i}$, a média de $Q_{i}$, e por fim, $Q$.

$$
\begin{aligned}
Q_{i}\left(P_{i}, P_{i, e}\right) & =\frac{1}{100} \sum_{e=1}^{100} Q_{e}\left(P_{i}, P_{i, e}\right), \\
Q\left(P, P_{e}\right) & =\frac{1}{N} \sum_{i=1} Q_{i}\left(P_{i}, P_{i, e}\right) .
\end{aligned}
$$

Em conformidade com a rede de referência apropriada, uma rede totalmente conectada implica $P=P_{e}$ possuindo um desequilíbrio mínimo e, assim, $Q=0$. Já uma rede esparsa, as probabilidades $P$ e $P_{e}$ diferem, o que indicará a presença de um alto desequilíbrio, daí, $Q \gg 0$. Observa-se que não faz sentido atribuir uma alta Complexidade a uma rede muito esparsa ou uma rede totalmente conectada, dessa forma, a utilização do divergente $Q$ ou da Entropia $S$ por si só, não é suficiente, com isso, a Complexidade Estatística foi proposta utilizando o produto dessas duas quantidades [Rosso et al. 2007].

$$
C(P)=Q\left(P, P_{e}\right) S(P) \in[0,1] .
$$

Observamos que para o caso de uma rede muito esparsa, $S(P)=0$ e $C(P)=0$. Já no caso de uma rede totalmente conectada $S(P)=1$, segue-se que $P=P_{e}$ que implica $Q\left(P, P_{e}\right)=0$, assim , $C(P)=0$. Com isso, temos a formação do plano ComplexidadeEntropia que pode ser utilizado para caracterização de Redes Complexas.

\section{Resultados e Discussão}

Com o propósito de verificar a consistência do modelo em sua capacidade de discernir Redes Complexas de acordo com sua topologia, foram avaliados grafos de Erdős-Rényi [Erdos 1959], Small World [Watts and Strogatz 1998], Scale-Free [Barabási and Albert 1999] e $k$-Regular, como ponto de partida para termos uma referência sólida dos seus comportamentos no plano $S \times C$, assim, comparamos aos resultados apresentados pelas redes veiculares, com variações na organização da rede em V2V, V2I e V2V2I. 


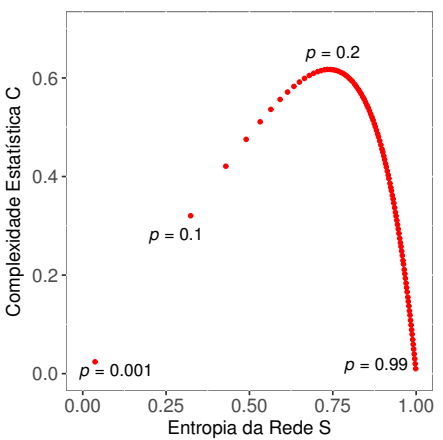

(a) Erdős-Rényi

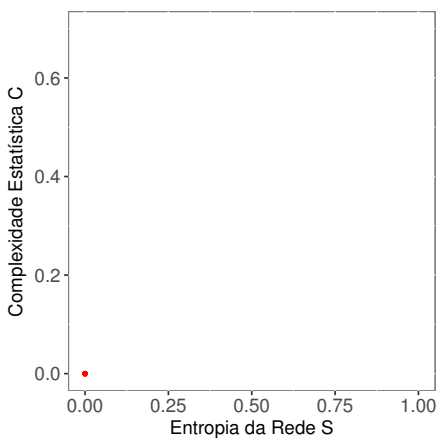

(b) $k$-Regular

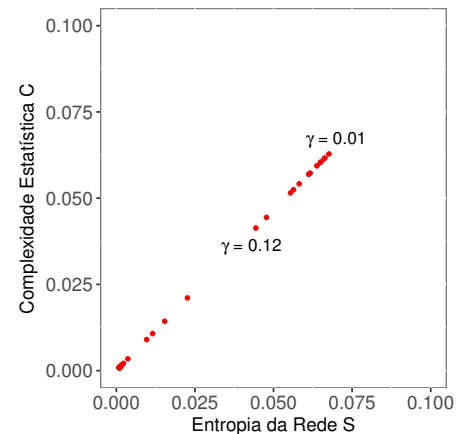

(c) Scale-Free

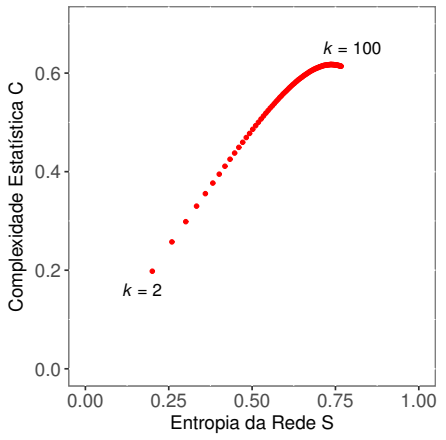

(d) Small-World

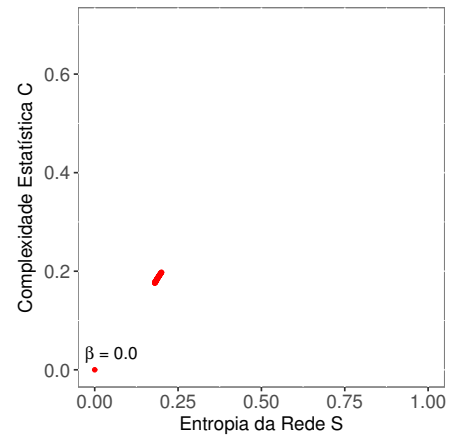

(e) Small-World $k=2$

Figura 1: Na figura (a), temos os resultados do Modelo Erdős-Rényi com $N=$ 1000 e uma variação a probabilidade de conexão $\rho$. Em (b), observamos o comportamento do grafo $k$-regular no plano para qualquer $k$. A figura (c) apresenta os resultados do modelo Scale Free de acordo com a variação de $\gamma$. Em (d) e (e), verificamos as características do grafo Small-World variando o grau médio $\boldsymbol{k}$ e a probabilidade de religação $\beta$.

\subsection{Redes Sintéticas}

Para gerar as redes de Erdős-Rényi utilizamos $N=1000$ e variamos sua probabilidade de conexão $p$, tal que, $p \in[0,1]$. Como pode ser visto na Figura 1a, a medida que $p$ aumenta, a Entropia segue crescendo, no entanto, a Complexidade apresenta um máximo em $p=0.2$ e a partir desse ponto vai diminuindo bruscamente. $\operatorname{Em} p=0.99$, temos que a Entropia $S \rightarrow 1$ e a $C \rightarrow 0$, o que sugere que temos uma rede muito conectada, porém com uma estrutura aleatória de acordo com os conceitos apresentados na Seção 2.4. Já em um grafo $k$-regular, $S=0$ e $C=0$ para qualquer $k$, como ilustrado na Figura $1 \mathrm{~b}$, onde foram gerados grafos regulares com $N=1000$ e $k \in[0, N-1]$ e não houve nenhuma alteração em seus resultados.

As redes Scale-Free foram geradas de acordo com o modelo [Barabási and Albert 1999], onde sua distribuição do grau $P(k)$ segue a lei da potência: $P(k) \sim k^{-\gamma}$, tal que $\gamma \in[0,10]$ e $N=1000$. A variação de $\gamma$ determina a velocidade do decaimento da distribuição do grau, de forma que quanto maior, maior a chance de termos grandes componentes conectados a maioria dos nós, aproximando-se de um grafo regular ou geométrico, assim, à medida que $\gamma \rightarrow \infty, S \rightarrow 0$. Verificando os resultados apresentados na Figura 1c, notamos que, em geral, os grafos correspondentes às redes Scale-Free apresentam $S<0.07$ e $C<0.07$, com uma transição mais brusca em $\gamma=0.12$. 


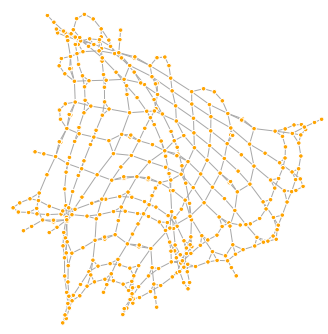

(a) $\mathrm{V} 2 \mathrm{~V}$

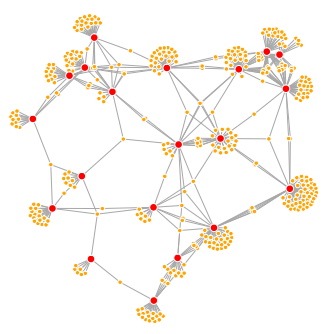

(b) V2I

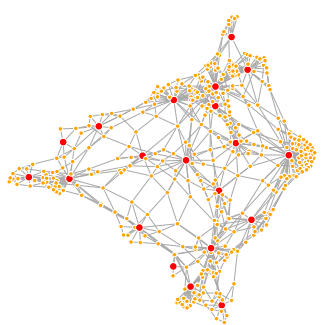

(c) V2V2I

Figura 2: Representação da topologia da rede Anaheim com $N=416$.

De acordo com os resultados apresentados na Figura 1d, podemos analisar o comportamento transitório das Redes Small-World. Com a variação do grau médio da rede inicial $k \in[2,100]$, temos uma mudança de característica na dinâmica da topologia da rede. Levando em consideração o modelo de criação do grafo Small-World, onde $k$ define o grau de cada nó, no grafo $k$-regular inicial, de onde partem as religações entre as arestas de acordo com a probabilidade de religação $\beta$. Contudo, a influência de $\beta$ nos resultados, não é tão relevante quanto o grau médio, analisamos $\beta \in[0,1] \operatorname{com} \beta=0$ e $k=2$, nos resta apenas um grafo regular como apresentado na Figura 1e já para os demais valores de $\beta$, as características da rede Small-World no plano de Complexidade e Entropia não apresentam variações tão relevantes em relação aos demais.

\subsection{Redes Veiculares}

Para as 17 redes reais descritas na Seção 2.1, realizamos o pré-processamento na rede de forma a adaptar de acordo com as definições da rede na Seção 2.2, removendo os laços e considerando uma rede não-direcionada. Calculando os quantificadores de Teoria da Informação, mapeamos os resultados no plano Complexidade-Entropia, onde essas redes na sua topologia original são consideradas redes V2V. Observando os resultados de uma rede V2V no plano de acordo com a Figura 3, identificamos um comportamento próximo das redes Small-World descrito na Figura 1e, mas com uma tendência de transição, aproximando-se de uma rede Scale-Free.

Com propósito de analisar a transição na topologia das redes veiculares, utilizamos os dados reais das redes, que caracterizam uma topologia V2V ilustrada na Figura 2a, para simular topologias V2I e V2V2I, observando a variação das características da rede à medida que modificamos a estrutura da rede real. Para criar uma rede com conexões V2I, foram removidas todas as conexões entre veículos, 5\% de nós de foram escolhidos de forma aleatória na rede e estabelecidos como infraestruturas. A determinação de escolher $5 \%$ dos nós como infraestruturas foi empírica, baseando-se na suposição que o número de infraestruturas presentes em uma VANET é muito menor que o número de veículos em geral. Então, para cada veículo escolhemos qual infraestrutura encontra-se mais próxima e estabelecemos a conexão V2I. Caso hajam duas infraestruturas com a mesma distância 
mínima, permite-se a conexão do veículo às duas, assumindo que em um cenário global, há grandes chances do veículo transitar entre o alcance de ambas.

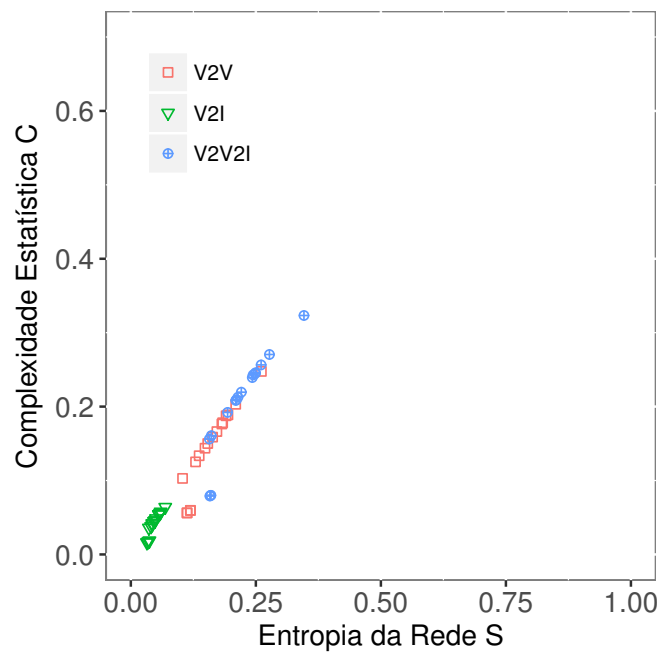

Figura 3: Plano Complexidade-Entropia com resultado da análise de todas as redes veiculares na topologia V2V, V2I e V2V2I.

Seus resultados no plano Complexidade-Entropia estão na Figura 3 e a estrutura da rede está ilustrada na Figura 2b. Em comparação aos resultados apresentados anteriormente, nos indicam uma semelhança das características da rede Scale Free com as Redes Veiculares em uma topologia V2I. Considerando o cenário V2V2I, as conexões existentes nas Redes Veiculares entre veículos foram mantidas, apenas adicionando as infraestruturas, do mesmo modo que em V2I (Figura 2c), conectando os nós veículos aos nós mais próximos que foram selecionados aleatoriamente como infraestruturas. Analisando o plano na Figura 3, observamos a transição das características das Redes Veiculares V2V2I com valores mais próximos do modelo Small-World, distanciando-se do Scale-Free, que em comparação aos resultados da Figura 1d, podemos notar que as redes V2V2I possuem um comportamento próximo das Redes Small-World com $k$ entre 2 e 10.

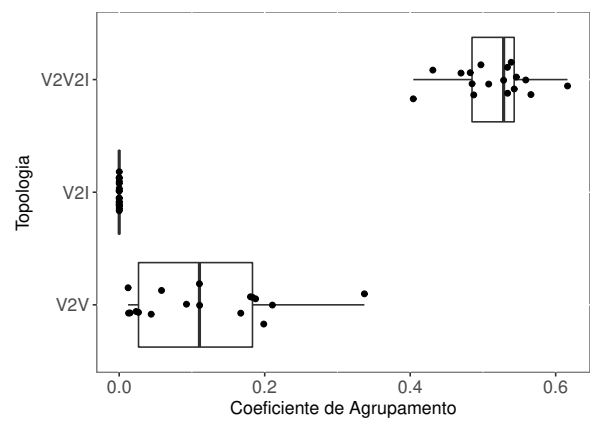

(a) Coeficiente de agrupamento

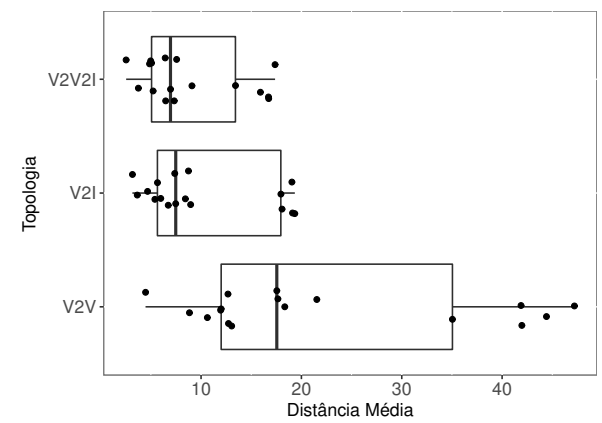

(b) Distância Média

Figura 4: Diagrama de caixa descrevendo a variação do Coeficiente de Agrupamento (a) e Distância Média (b) de acordo com a mudança de topologia para cada Rede Veicular. 
Para uma verificação mais detalhada desse comportamento, recorremos a métricas clássicas de redes complexas como Coeficiente de Agrupamento e Distância Média, onde redes Watts-Strogatz apresentam um baixo valor de distância média e um alto coeficiente de agrupamento. Podemos observar nas Figuras 4 e 4 a que a mudança de topologia $\mathrm{V} 2 \mathrm{~V} \rightarrow \mathrm{V} 2 \mathrm{I}$, remove a presença de grupos, anulando o coeficiente de agrupamento. Da mesma forma, em V2V $\rightarrow$ V2V2I, observamos que o coeficiente de agrupamento aumenta e a distância média na rede diminui, como ilustrado na Figura 4b, indicando um comportamento Small-World na rede. Diante disso, por indução, supomos que o aumento do número de infraestruturas na rede deve aumentar a presença de agrupamentos e diminuir a distância média ainda mais, já que teremos uma maior quantidade de nós intermediários com um alto valor de $k$ aumentando a conectividade da rede.

\section{Conclusão}

$\mathrm{Na}$ abordagem de Teoria da Informação apresentada, conseguimos utilizar um método bem sucedido em caracterizar diferentes tipos de redes reais, com o propósito de caracterizar Redes Veiculares no contexto dos modelos de Redes Complexas. Assim, observamos que Redes Veiculares com uma topologia V2I, apresentam estruturas semelhantes ao grafo Scale-Free, com coeficiente de agrupamento nulo, onde as infraestruturas possuem um comportamento de $h u b$. Já as topologias V2V e V2V2I, são melhores descritas pelo modelo Small-World, com algumas variações na sua estrutura, tendo em vista que a presença de infraestruturas induzem a existência de hubs na rede, consequentemente temos um coeficiente de agrupamento maior em V2V2I e uma distância média menor.

A identificação das características dessas topologias são relevantes para definição de protocolos de roteamento e disseminação de dados adequadamente, redes com alto coeficiente de agrupamento indicam uma maior tolerância à falhas, por possuir menos chance de apresentar links críticos capazes de conectar ou desconectar grandes componentes, e maior probabilidade de um nó encontrar um caminho alternativo a um nó vizinho caso a conexão existente seja extinta. Uma baixa distância média aponta para uma maior facilidade de um nó se comunicar com outro nó qualquer nessa rede, dessa forma, a disseminação de dados na rede pode ocorrer de forma mais rápida. Daí, podemos concluir que a topologia V2V2I é mais robusta em relação à V2V e V2I, sendo mais capaz de lidar com falhas e de distribuir a informação de forma mais eficiente na rede. Para os próximos trabalhos, devemos analisar o impacto mais detalhado do aumento da quantidade de infraestruturas na rede e aprofundar na variação das características ao longo do tempo, com base em dados de deslocamento e velocidade, em busca de uma caracterização mais abrangente utilizando este método em comparação as demais abordagens existentes na literatura.

\section{Referências}

[Aquino et al. 2015] Aquino, A. L., Cavalcante, T. S., Almeida, E. S., Frery, A. C., and Rosso, O. A. (2015). Characterization of vehicle behavior with information theory. The European Physical Journal B, 88(10):257.

[Barabási and Albert 1999] Barabási, A.-L. and Albert, R. (1999). Emergence of scaling in random networks. science, 286(5439):509-512.

[Erdos 1959] Erdos, P. (1959). On random graphs. Publicationes mathematicae, 6:290-297. 
[Hajlaoui et al. 2016] Hajlaoui, R., Guyennet, H., and Moulahi, T. (2016). A survey on heuristic-based routing methods in vehicular ad-hoc network: Technical challenges and future trends. IEEE Sensors Journal, 16(17):6782-6792.

[Liang et al. 2015] Liang, W., Li, Z., Zhang, H., Wang, S., and Bie, R. (2015). Vehicular ad hoc networks: architectures, research issues, methodologies, challenges, and trends. International Journal of Distributed Sensor Networks, 11(8):745303.

[Liu et al. 2016] Liu, J., Wan, J., Wang, Q., Deng, P., Zhou, K., and Qiao, Y. (2016). A survey on position-based routing for vehicular ad hoc networks. Telecommunication Systems, 62(1):15-30.

[Lopez-Ruiz et al. 1995] Lopez-Ruiz, R., Mancini, H. L., and Calbet, X. (1995). A statistical measure of complexity. Physics Letters A, 209(5-6):321-326.

[Newman 2005] Newman, M. E. (2005). A measure of betweenness centrality based on random walks. Social networks, 27(1):39-54.

[Pappalardo et al. 2013] Pappalardo, L., Rinzivillo, S., Qu, Z., Pedreschi, D., and Giannotti, F. (2013). Understanding the patterns of car travel. The European Physical Journal Special Topics, 215(1):61-73.

[Rosso et al. 2007] Rosso, O., Larrondo, H., Martin, M., Plastino, A., and Fuentes, M. (2007). Distinguishing noise from chaos. Physical review letters, 99(15):154102.

[Tang et al. 2016] Tang, J., Liu, F., Zhang, W., Zhang, S., and Wang, Y. (2016). Exploring dynamic property of traffic flow time series in multi-states based on complex networks: Phase space reconstruction versus visibility graph. Physica A: Statistical Mechanics and its Applications, 450:635-648.

[Watts and Strogatz 1998] Watts, D. J. and Strogatz, S. H. (1998). Collective dynamics of 'small-world' networks. nature, 393(6684):440.

[Wiedermann et al. 2017] Wiedermann, M., Donges, J. F., Kurths, J., and Donner, R. V. (2017). Mapping and discrimination of networks in the complexity-entropy plane. Physical Review E, 96(4):042304.

[Yan et al. 2017] Yan, Y., Zhang, S., Tang, J., and Wang, X. (2017). Understanding characteristics in multivariate traffic flow time series from complex network structure. Physica A: Statistical Mechanics and its Applications, 477:149-160.

[Yousefi et al. 2006] Yousefi, S., Mousavi, M. S., and Fathy, M. (2006). Vehicular ad hoc networks (vanets): challenges and perspectives. In ITS Telecommunications Proceedings, 2006 6th International Conference on, pages 761-766. IEEE.

[Zhang et al. 2013] Zhang, D., Huang, H., Zhou, J., Xia, F., and Chen, Z. (2013). Detecting hot road mobility of vehicular ad hoc networks. Mobile Networks and Applications, 18(6):803-813. 